\section{TRES PREMISAS NOCIVAS EN EL ESTUDIO DEL GUETO NORTEAMERICANO*1}

Loïc J.D. Wacquant ${ }^{2}$

El artículo desarrolla una crítica profunda a los abordajes que predominan en el estudio de la pobreza urbana en los Estados Unidos. A juicio del autor, tales abordajes simplifican al extremo la noción de gueto, convirtiéndola en el equivalente de bolsón de pobreza, despojándola así de su especificidad histórica, su potencial heurístico y su valor sociológico. El autor aboga por la recuperación de una perspectiva institucionalista de la noción de gueto, así como por romper con el exotismo que se le atribuye en la actualidad, de modo de mostrar que el gueto no es el resultado de un particularismo local ni de una determinada esencia cultural propia a sus habitantes, sino que el resultado complejo de la articulación de una serie de agentes

\section{THREE PERNICIOUS PREMISES IN THE STUDY OF THE AMERICAN GHETTO*1}

Loïc J.D. Wacquant ${ }^{2}$

The paper develops a deep criticism of the dominant approaches to the study of urban poverty in the United States. According to the author, these approaches simplify the notion of ghetto to the extreme, thus turning this concept into the equivalent to pocket of poverty and divesting the term of historical specificity, heuristic potential and psychological value. The author advocates for the recovery of an institutional approach to the notion of ghetto and for the end to the exoticism built around this concept; thus, it is argued that the ghetto is the complex result of the articulation of a series of institutional agents and factors along with the operation of dominant political and economic structures. These agents and structures 
y factores institucionales y del funcionamiento de estructuras de dominación política y económica que trascienden al gueto y atraviesan a la sociedad en su conjunto. El artículo pretende ser un primer paso en esta dirección.

PALABRAS CLAVE: GUETO, POBREZA URBANA, MARGINALIDAD, SOCIOLOGÍA URBANA. transcend the ghetto and reverberate throughout society rather than being the result of local particularities or the cultural essence of its dwellers. The paper aims to lay the foundations for further research.

KEYWORDS: GHETTO, URBAN POVERTY, SOCIAL MARGINALITY, URBAN SOCIOLOGY.
* Varios amigos y colegas han tenido la gentileza de hacer comentarios en sucesivas versiones de este trabajo. Entre todos ellos menciono especialmente a Bill Wilson, quien me introdujo en esta materia y me animó a afrontar estos temas incluso cuando no estaba de acuerdo conmigo en cómo los abordaba Godfried Engbersen, Neil Fligstein, Martin Sánchez-Jankowski, Claude Fischer y Chris Pickvance por brindar sugerencias que me obligaron a aclarar mis argumentos incluso cuando me resistía a hacerlo y Wilhelm Heitmeyer y Federico Neiburg, quienes propiciaron el incentivo para la publicación en alemán y portugués de versiones previas de este trabajo. También se agradece el apoyo de la Fundación Russell Sage, lugar donde se escribió el bosquejo inicial de esta publicación.

1 Nota del editor: Artículo publicado originalmente en International Journal of Urban and Regional Research (Vol. 21, №2, Junio 1997). Esta versión en español ha sido autorizada por el autor en el marco de su visita a la Universidad de Chile de octubre de 2013.

2 Estados Unidos. Ph. D en sociología. Profesor de sociología de la Universidad de California en Berkeley e Investigador del Centro Europeo de Sociología, en París.
* Many friends and colleagues have been kind enough to comment on successive versions of this article. Among them a special mention is due to Bill Wilson, who drew me to the topic in the first place and encouraged me to confront these issues even when he disagreed with how I framed them; Godfried Engbersen, Neil Fligstein, Martin Sánchez-Jankowski, Claude Fischer and Chris Pickvance for pointed suggestions that forced me to clarify my arguments even as I resisted them; and Wilhelm Heitmeyer and Federico Neiburg who supplied the impetus for earlier versions in German and Portuguese. The support of the Russell Sage Foundation, where the initial draft of this paper was written, is gratefully acknowledged.

1 Editorial note: Paper published in International Journal of Urban and Regional Research (Vol. 21, №2, June 1997). This Spanish version has been authorized by the author on the occasion of his visit to the University of Chile in October 2013.

2 United States. Sociology Ph.D. Professor of Sociology, University of California at Berkeley. Researcher at the Centre Européen de Sociologie et de Science Politique, París. 
Ahora, ¿qué fue lo que llevó al hombre a considerar estas proposiciones mitológicas o creencias como verdaderas? ¿Tal vez un enfrentamiento con una realidad determinada? De ninguna manera... Son nuestras ideas, nuestras creencias, las que le otorgan realidad a los objetos de pensamiento (Emile Durkheim, Pragmatisme et sociologie, 1913).

Tres inclinaciones o premisas, profundamente arraigadas, han dominado el reciente debate sobre división racial y pobreza urbana en los Estados Unidos. Estas premisas tienen sus raíces en antiguas nociones que encasillan a los pobres, en especial a los pobres de raza negra, como personas de dudosa moral y ven a la ciudad como un lugar nefasto que interfiere y corrompe la vida social, particularmente en el caso de las clases más bajas $^{3}$. Dotadas de plausibilidad gracias al peso de la historia y a la inercia intelectual, y reforzadas por un lenguaje nacionalista de corte individualista que minimiza a las clases y disfraza el concepto de dominación etnoracial, estas premisas se alzan como las piedras angulares del presente doxa académico sobre este tema y, por tanto, son obje-

3 Boyer, 1978; Katz, 1983; Patterson, 1986; Fishman, 1988 to de pocas discusiones o cuestionamientos. ${ }^{4}$ Sin embargo, estos principios truncan y distorsionan nuestro entendimiento sobre la actual (re)articulación del color, clase y lugar que está ocurriendo en la metrópolis norteamericana.

La primera y más reciente tendencia es la dilución de la noción de gueto, la cual es utilizada para referirse simplemente a un área urbana de pobreza generalizada e intensa, creando confusión en torno a la base racial y carácter de este tipo de pobreza y despojando esta noción original de su significado histórico y contenido sociológico. El segundo de estos principios data desde hace un siglo y concibe al gueto como una formación social "desorganizada" que puede ser completamente analizada en términos de carencias y deficiencias (sean estas individuales o colectivas) dejando de lado la identificación de los principios que subyacen bajo su orden interno y rigen su modo específico de funcionamiento. La tercera premisa, cuyo origen viene del concepto de desorganización, es la tendencia a presentar al gueto y sus habitantes como

4 Un gran número de publicaciones se pueden citar aquí. Basta mencionar el trabajo de Jencks y Peterson The Urban Underclass (1991), el cual reúne ejemplos paradigmáticos de cada una de estas premisas. Esto no quiere decir que la literatura de las ciencias sociales enfocada en temas raciales o pobreza urbana sea monolítica (para acceder a estudios más amplios, véase Wilson y Aponte, 1985; Marks, 1991; y Devine y Wright, 1993), sino que las variaciones que presenta se encuentran en gran parte incluidas dentro del espacio analítico delimitado por estos tres preconceptos.

revista invi № 79 / Noviembre 2013 / Volumen №28: 165-187 167 
algo exótico, destacando los aspectos más extremos e inusuales de la vida en estas áreas desde una perspectiva exterior y superior; se trata de una visión desde el punto de vista de los dominantes. Cada una de estas premisas está asociada a una serie errores y descuidos analíticos tan comunes que pasan desapercibidos o, peor aún, llegan a ser parte del fenómeno mismo. Juntas, estas premisas forman un enorme "obstáculo epistemológico" a la teóricamente rigurosa y empíricamente precisa sociología sobre conflictos raciales y marginalidad urbana en los Estados Unidos de hoy puesto que ellas convergen para ocultar la división tanto entre la percepción del sentido común y el análisis científico social como entre las controversiales y complejas realidades que deben ser explicadas y lo que la gente desea creer sobre ellas ${ }^{6}$. El presente estudio sobre estas tres nocivas premisas se ofrece como un prolegómeno a este tipo de sociología.

5 Bachelard, 1938.

6 Tal como lo mencionó Wittgenstein (1977: 17): “lo que hace que un texto sea difícil de comprender -si es significativo e importante- no es el que tengas que ser un erudito en temas complejos antes de abordarlo, sino que es el contraste entre comprender el tema y lo que las personas quieren ver. A raíz de esto, las cosas que parecen más obvias pueden convertirse en las más difíciles de entender. Lo que debe superarse es la dificultad relacionada con la voluntad y aquella vinculada al intelecto".

\section{Recuperando la concepción institucionalista del gueto}

Las últimas discusiones sobre temas raciales y pobreza en los barrios deprimidos de la ciudad norteamericana muestran una tendencia a relacionar al gueto con cualquier área que presente altos niveles de pobreza, independiente de su población y composición social. Paul Jargowski y Mary-Jo Bane ${ }^{7}$ ofrecen un ejemplo de esta omisión de la dimensión racial e institucional en la noción de gueto:

"Definimos gueto como un área donde la tasa de pobreza promedio dentro de una zona censal mayor al 40\%. El gueto negro corresponde, entonces, a aquellas personas de escasos recursos, de cualquier raza o grupo étnico, que se ubiquen dentro de esta zona censal de alta pobreza... Diversas visitas a distintas ciudades confirmaron que este criterio del $40 \%$ estuvo cerca de identificar áreas que presentaban características de guetos de acuerdo a sus condiciones de vivienda. Más aún, las áreas seleccionadas bajo este criterio coincidieron con los barrios que funcionarios municipales y de la Oficina del Censo consideran como guetos... Es importante separar nuestra definición de gueto, elaborada a partir de

\footnotetext{
7 Jargowski y Bane $(1991$, p. 239, 241) énfasis añadido
} 
criterios de pobreza, de aquella basada en composición racial. De acuerdo a nuestra proposición, no todos los tramos compuestos por gente de raza negra corresponden a guetos ni todos los guetos están conformados por gente de raza negra".

La (re)definición de este término merece ser citada en extenso ya que incluye prácticamente todos los aspectos negativos que han marcado el uso de este concepto: (1) es totalmente arbitrario (tal como sus autores lo reconocen en la página 239): ipor qué excluir zonas rurales o suburbanas y utilizar tramos censales como fuente de recolección de datos, hacer uso de la "línea de pobreza" como barra de medición y fijar la tasa del $40 \%$ de personas de escasos recursos como punto de corte? (2) Carece de base sociológica puesto que está ligado al ingreso familiar (indicador poco fiable en encuestas normalizadas, especialmente entre poblaciones que cuentan con empleos irregulares) y al aspecto de las viviendas, dejando de lado las relaciones sociales y económicas que lo determinan; (3) aparenta estar libre de prejuicios raciales cuando en realidad se refiere únicamente a enclaves urbanos habitados por familias negras de escasos recursos, excluyendo las áreas dominadas por blancos pobres; (4) es burocrática y derivada de categorías administrativas, esto se debe a que la viabilidad de este concepto radica en la existencia y disponibilidad de datos provenientes de entes gubernamentales tales como la Oficina del Censo y su definición de "área de pobreza" y (5) mezcla sin reparos un concepto histórico-analítico con nociones concebidas por las elites municipales y estatales ("lo que los funcionarios municipales y miembros de la Oficina del Censo consideran gueto") sin dar oportunidad alguna para analizar lo que estas percepciones de sentido común pueden significar respecto a la definición del objeto que está siendo estudiado ${ }^{8}$.

Esto supone olvidar que (a pesar de ser medidas) gran parte de las áreas urbanas de "extrema pobreza" en la Franja de la Herrumbre norteamericana son herederas directas de los antiguos "cinturones negros". Decir que estas áreas son guetos solo por carecer de recursos significa revertir la causalidad social e histórica: el hecho de que estas áreas fueran y sigan siendo guetos explica el inusual y persistente grado de faltas de oportunidades laborales y miseria y no lo contrario. Clasificar como gueto a cualquier área que muestre una alta tasa o concentración de pobreza no solo es una acción arbitraria y empíricamente problemática; se está hablando de despojar a este término de su significado histórico y borrar su trascendencia sociológica, creando así obstáculos para investigaciones enfocadas en los

8 Todas estas concepciones erróneas se repiten y extienden en el trabajo de Jargowski Poverty and Place: Ghettos, Barrios, and the American City, donde se equipara efectivamente el proceso de guetización con el deterioro urbano.

revista invi №79 / Noviembre 2013 / Volumen № 28: 165-187 169 
criterios y procesos donde la exclusión toma un papel protagónico. De esta manera se oculta el hecho de que la población de raza negra sea el único grupo en la historia de los Estados Unidos que ha experimentado la guetización, siendo este un proceso involuntario, permanente y de una marginación residencial total centrada en las castas como la base para el desarrollo de una estructura social paralela (e inferior) ${ }^{9}$.

De acuerdo a los orígenes históricos y al uso historiográfico del término ${ }^{10}$, un gueto no es simplemente una entidad topográfica o un conjunto que reúne a familias e individuos de escasos recursos sino que es más bien una forma institucional, una unión espacial de mecanismos de confinamiento y

9 Los trabajos de Spear (1968), Osofsky (1971), Kusmer (1976), Zunz (1982), Farley y Allen (1987), Jaynes y Williams (1989), Hirsch (1993) y Massey y Denton (1993) documentan ampliamente la singularidad, intensidad y persistencia de la segregación y exclusión institucional de la población negra en el transcurso del siglo de vida del gueto.

10 Wirth, 1928: 11-62; Cooperman y Curiel, 1990. control etnoracial ${ }^{11}$ históricamente determinada ${ }^{12}$. En términos de una tipificación ideal, un gueto puede ser descrito como una formación socioespacial reducida que presenta una uniformidad racial y/o cultural determinada por (1) la relegación forzosa de (2) una población "estereotipada negativamente"13, como lo fue el caso de los judíos en la Europa medieval o los afroamericanos en los Estados Unidos de hoy, a (3) un "territorio fronterizo"14 donde esta población (4) desarrolla bajo represión una serie de instituciones paralelas que actúan como sustitutos funcionales o como protección contra las entidades dominantes de la sociedad $^{15}$, (5) duplicando a estas últimas en un nivel incompleto e inferior y (6) manteniendo a

11 Wacquant, 1991

12 En su clásica revisión histórica, Louis Wirth (1964:84-85) insiste en que el gueto es tanto "un instrumento de control" como una "forma de albergue mediante la cual una minoría ha sido efectivamente sometida por un grupo dominante". Sin embargo, este autor comete un serio error en referirse a la guetización como un "proceso natural" que afecta a "cada persona y a cada grupo cultural". Para obtener una descripción más acabada sobre los principios básicos del institucionalismo (y neoinstitucionalismo) dentro de la tradición sociológica, véase DiMaggio y Powell (1992). Más detalles sobre el concepto de confinamiento y su uso en las teorías (neo)Weberianas respecto a la desigualdad de clase y grupo pueden ser encontrados en Parkin (1979), Murphy (1987), Brubaker (1991: Parte 1), y Manza (1992).

13 Weber, 1978: 385-7

14 Hogan, 1980

15 Spear, 1968; Meier y Rudwick, 1976: 232-70 
quienes confían en tales instituciones paralelas en un estado de dependencia estructural ${ }^{16}$. Visto de otro modo, el gueto es una formación etnoracial que combina e inscribe dentro de la objetividad del espacio y de las instituciones propias del grupo las cuatro "formas elementales" más importantes de dominación racial, a saber, categorización, discriminación, segregación y violencia basada en la exclusión ${ }^{17}$.

El hecho de que los guetos sean sitios donde la miseria ha sido un mal endémico no significa que sean lugares pobres o que presenten carencias. Sin duda, el "Bronzeville" de los años 1940 era más próspero que las comunidades negras ubicadas al sur del país y concentraba quizás a la burguesía afroamericana más numerosa y opulenta de su época ${ }^{18}$. Por otra parte, y a pesar de su pobreza extrema, no todas las áreas de bajos ingresos entran en la clasificación de gueto. Las ciudades en declive de población blanca de las zonas desindustrializadas tanto del medio oeste como de los Apalaches, las comunidades

16 Weaver, 1948; Fusfeld y Bates, 1984; Logan y Molotch, 1987.

17 Wacquant, 1995.

18 Drake y Cayton, 1962; para un análisis más amplio consúlteseTrotter, 1993. rurales deprimidas del delta del Misisipi, las reservas de nativos americanos y los barrios ${ }^{19}$ pobres de la región suroeste (sin mencionar a vastos sectores de los Estados Unidos durante la Gran Depresión) no muestran el patrón organizacional del "gueto negro": ellas no son, y nunca han sido, "colonias de carácter filántropo, económico, empresarial e industrial" ${ }^{20}$ de la sociedad blanca ${ }^{21}$.

Una aproximación institucionalista (o relacional) al concepto de gueto no solo es más coherente con el uso y origen histórico de este término. Aquí se cuestiona y pone en primer plano las variables que enfoques de corte más nominalista y escalonado ven como condiciones secundarias que no requieren mayor estudio tales como la división racial, cuyos efectos estructurales pueden variar con el paso del tiempo incluso si las tasas de medición se mantienen iguales, la ineficacia y débil presencia de instituciones de servicios públicos que no son más que elementos organizacionales estandarizados

19 Barrio de hispanohablantes en una ciudad norteamericana.

20 Clark, 1965; ver también Connolly, 1977.

21 Para obtener una descripción sobre la ubicación y estructura ecológica, social e institucional de los barrios latinos pobres que marcan una diferencia con las urbes negras dentro de la metrópolis norteamericana, véase Moore y Pinderhughes (1993). Establecer si las tribus de nativos americanos son un subtipo de gueto u otra clase de mecanismo de sometimiento etnoracia requiere una discusión que en este caso no es posible; Snipp (1986) y Cornell (1990) ofrecen información que puede dar una respuesta a esta interrogante.

revista invi № 79 / Noviembre 2013 / Volumen № 28: 165-187 171 
de otros barrios, el excesivo papel desempeñado por la policía e instituciones penales y la ausencia de un "mecanismo de contravalor indígena" 22 . Específicamente, se problematiza lo que la perspectiva lineal y "demográfica" da por sentado: las bases y medios a través de los cuales se determina la relegación dentro del gueto. Así, en vez de situar este último punto de vista junto a distribuciones continuas de ingreso, vivienda, segregación o pobreza a nivel vecinal, un enfoque institucionalista busca identificar roturas al interior del tejido urbano y hacer un seguimiento a la (re)configuración de las líneas divisorias que tienen al gueto como su manifestación física. ${ }^{23}$

En este sentido, las fronteras, forma, composición interna, vínculos externos y soporte estructural de este territorio de exclusión se transforman en interrogantes esenciales que deben ser respondidas mediante análisis empíricos y

22 Logan y Molotch, 1987: 131; Davis, 1991: 304-9.

23 Para un estudio de referencias sobre la producción sociopolítica de disparidades urbanas enmarcada dentro de un proceso institucional (y no ecológico), véase la reconstrucción del "apartheid urbano de Marruecos" y la transmutación de la división de castas en clases sociales en el Rabat postcolonial propuesta por Abu-Lughod (1980). Véase también la brillante reconstrucción de Hirsch (1983) del fundamental rol desempeñado por entes locales y federales en la creación del "Segundo Gueto" de Chicago entre las décadas de 1940 y 1960. no a través de juegos de definiciones. Cada una de las características que forman parte del tipo ideal de gueto recién esbozado (limitación, estigma, separación y finitud territorial, diferenciación y paralelismo institucional, duplicación y dependencia funcional), así como el grado de "vinculación" entre ellas, pueden convertirse en variables que pueden ser medidas con precisión.

\section{Abandonando el concepto de "desorganización"}

El antropólogo Arjun Appadurai ${ }^{24}$ demostró que ciertos lugares son cada vez más frecuentemente descritos y discutidos a través del uso "tropos", esto es, una serie recurrente de imágenes y estrategias narrativas que predeterminan y distorsionan la forma en que estas áreas son percibidas y conceptualizadas. En la sociedad norteamericana y en las ciencias sociales, el

$24 \quad$ Appadurai, 1988. 
"tropo" con el que se ha caracterizado al gueto desde sus orígenes a finales del siglo XIX se conoce como "desorganización". ${ }^{25}$

Desde la antigua escuela de ecología humana de Chicago, pasando por los estudios de la crisis urbana de los años 1960, hasta las últimas investigaciones sobre el surgimiento de la llamada "subclase" y sus temibles implicaciones ${ }^{26}$, los analistas dan por establecido el hecho de que el gueto puede ser satisfactoriamente analizado en términos privativos mediante la identificación de sus deficiencias, y las de sus habitantes, y la descripción de cómo (y cuánto) ambas se separan

25 Ward (1989) ofrece un extenso análisis sobre la continuidad y cambios en la caracterización social de barrios marginales, guetos y otros territorios de relegación urbana en la era de la expansión capitalista industrial. Geoffrey Biddle (1992), en su trabajo Alphabet City, proporciona una vívida y actualizada representación fotográfica del enfoque basado en la "desorganización" tal como se observa en Loisada, el barrio puertorriqueño ubicado en la parte baja del este de Nueva York. Camilo Vergara (1995) proporciona una valiosa rectificación respecto a este monocromático escenario al presentar un retrato matizado de los distintos tipos de "guetos" (aquí el autor utiliza la forma más común de este término, describiéndolo como un enclave segregado y en deterioro): los "guetos verdes" reclamados por la naturaleza, los "guetos institucionales" que concentran las indeseables "viviendas pobres del siglo XXI" (antros de droga y centros de rehabilitación, albergues para vagabundos, comedores sociales, cárceles, etc.) y los dinámicos y fluidos "nuevos guetos de inmigrantes".

26 Park y Burgess, 1925; Wirth, 1928; Shaw y McKay, 1942; Frazier, 1949; Banfield, 1970; Wilson, 1987; Anderson, 1991; Jencks y Peterson, 1991; Harrell y Peterson 1992. de la sociedad "establecida" al ser medidas de por supuestos estándares de "clase media". Generalmente, estas categorías de sociedad "establecida" y "clase media" se dejan sin definir con el propósito de expandir sus fronteras y de esta manera hacerlas calzar dentro de necesidades analíticas e ideológicas. ${ }^{27}$ Así entonces, el gueto es representado como un lugar de desorden y carencias, un espacio de caos, desviación, anomia y atomización, lleno de comportamientos que ofenden los preceptos de la moralidad y propiedad ya sea mediante excesos (aquellos relacionados con la delincuencia, la sexualidad y la fertilidad) o ca-

27 Un uso representativo de esta dualidad de sentido común se encuentra en la digresión de Christopher Jencks (1991, p. 215-218) sobre la "subclase contra las madres tradicionales". Este autor admite que si bien "existe un acuerdo general en reconocer el concepto de 'subclase' como un antónimo de 'clase media', o incluso de clase 'tradicional' (término que reúne a la clase media y a la clase trabajadora)", "nunca ha existido un consenso respecto a qué significa pertenecer a estas clases". De esta manera los conceptos clave de su análisis sobre el "crecimiento de la subclase norteamericana" están empañados por confusiones epistemológicas e indeterminaciones semánticas (incluyendo un gran número de alusiones morales). Jencks confiesa que la conformidad con la norma académica es su única justificación para utilizar esta noción mitad académica, mitad periodística: “En mi opinión, los cambios sociales no son suficientes para justificar la substitución del término subclase por el de clase baja. Sin embargo, como todos están hablando de subclase en vez de clase baja, yo haré lo mismo" (Ibíd.: 28). 
rencias (como lo es el caso del trabajo, el ahorro y la familia). ${ }^{28}$

Este concepto erróneo (en un sentido tanto representativo como epistemológico) está profundamente enraizado en las ciencias sociales norteamericanas. La historiadora Alice O'Connor ${ }^{29}$ ha demostrado que el marco asimilador de la escuela de Chicago constantemente habló de "la pobreza, la 'desorganización' y la segregación social como resultados inevitables" de los cuasi biológicos "procesos de crecimiento de la ciudad", omitiendo cuidadosamente tanto las estrategias de los empleadores como el "papel que desempeñan los políticos y el gobierno local en la creación y mantención de los guetos". E. Franklin Frazier, el primer afroamericano en dirigir el departamento de sociología de la Universidad de Chicago, utilizó el concepto de desorganización para referirse a

28 Goldfield y Lane (1973: 4-5) sostienen que "la patología del gueto ha actuado como una anomalía constante que empaña los ideales de la vida norteamericana". "El gueto es símbolo de pobreza en un país rico, discriminación en una nación donde todos son iguales, enfermedad en un país tecnológicamente avanzado y delincuencia en una sociedad regida por la ley".

29 O'Connor, 1995.

174 revista invi № 79 / Noviembre 2013 / Volumen № 28: 165-187 la urbanización negra del área norte de la ciudad. Su trabajo The Negro Family in Chicago ${ }^{30}$ se centra en la ruptura conyugal, decadencia moral, pobreza material, delincuencia y vicios de los que es presa la población "negra" en su migración a la metrópolis industrial. Resulta revelador que este académico elevara la estructura familiar al rango de indicador de desorganización social entre la comunidad afroamericana, anticipándose medio siglo a las preocupaciones y estrategias definidas por su sucesor William Julius Wilson ${ }^{31}$ en su trabajo The Truly Disadvantaged ${ }^{32}$.

Concebir al gueto como una forma institucional en vez de una acumulación de "patologías" permite reconocer que esta estructura no sufre de "desorganización social", un concepto cargado de mora$\operatorname{lidad}^{33}$ que debe desaparecer del léxico de la sociología a pesar de su ilustre pedigrí intelectual. Tal

30 Frazier, 1931.

31 Wilson, 1987.

32 La investigación a gran escala, interdisciplinaria, multimétodo y empírica dirigida por Wilson entre 1985 y 1990, destinada a expandir y completar el "bosquejo teórico" expuesto en The Truly Disadvantaged llevó por nombre "Proyecto sobre pobreza urbana y estructura familiar". Este trabajo necesitó de importantes recursos para analizar las correlaciones e implicaciones de la (des)composición familiar en distintas "áreas pobres". Cabe señalar de todos modos que la concepción de Wilson sobre la "disolución" familiar no es un factor causal independiente, sino que una variable interviniente que revela, transmite y amplifica los nefastos efectos de la precaria situación (masculina) dentro del mercado laboral.

33 Véase Wirth, 1964: p. 44-49. 
como William Foote Whyte ${ }^{34}$ sugirió medio siglo atrás en su clásico estudio sobre "las sociedades de las esquinas" del barrio italiano de Boston, lo que un observador externo ve como desorganización social es generalmente una forma distinta de organización social si uno analiza el problema de cerca. En este sentido, un estudio detallado de primera mano es lo que precisamente hace falta en el caso del gueto negro norteamericano.

De los 27 autores que participaron en The Urban Underclass (1991), un espléndido trabajo financiado y promocionado por un consorcio formado por importantes fundaciones filantrópicas y de investigación y que afirma ofrecer "las mejores y más actualizadas investigaciones y opiniones sobre el tema", sólo uno realizó un estudio en terreno sobre el gueto. Asimismo, en la conferencia celebrada en torno a esta "obra de referencia", el único antropólogo invitado a este evento abordó la problemática de la "subclase" desde un punto de vista etnográfico y teórico-cultural que se aleja de la discusión presente en el libro ${ }^{35}$. Debido a que la investigación sobre pobreza es dominada por

34 Whyte, 1943: 273, énfasis añadido.

35 Su nombre ni siquiera es mencionado en la lista de participantes y panelistas (Jencks y Peterson, 1991: v-vi) y su publicación está publicada en otro sitio (Newman, 1991). economistas, demógrafos, sociólogos que recurren a información derivada de censos y encuestas, trabajadores sociales y expertos en política social (o eruditos) quienes dominan esta disciplina; por esta razón, los estudios antropológicos sobre este tema son normalmente ignorados o, en el mejor de los casos, mencionados selectivamente para desempeñar una función estrictamente ornamental.

De forma extraordinaria, solo un puñado de estudios en terreno sobre la vida en los barrios deprimidos de las ciudades negras han aparecido desde las revueltas raciales de mediados de la década de 1960 y los pocos que han logrado ser publicados son generalmente ignorados -pienso en el brillante libro Runnin' Down Some Lines de Edith Folb ${ }^{36}$, sólo por mencionar uno. El resultado de esto son las mismas "clásicas" monografías que datan de la era de la guerra contra la pobreza, encabezadas por el cuarteto compuesto por Oscar Lewis ${ }^{37}$, Elliott Liebow $^{38}$, Ulf Hannerz ${ }^{39}$ y Lee Rainwater ${ }^{40}$, y que continúan siendo ritualmente citadas -e incluso caricaturizadas al extremo para reflejar preocupa-

36 Folb, 1980.

37 Lewis, 1966.

38 Liebow, 1967.

39 Hannerz, 1969.

40 Rainwater, 1970. 
ciones actuales ${ }^{41}$ - como si las bases, estructuras y dinámicas de la vida en el gueto (sub)proletariado estuviesen congeladas en el tiempo, presentando patrones idénticos a lo largo de tres décadas de enormes cambios a nivel económico, espacial y sociopolítico ${ }^{42}$.

Una revisión basada en la observación directa opuesta a mediciones poco exploratorias y completamente inadecuadas para estudiar la vida de poblaciones marginadas- revela inmediatamente que, lejos de ser desorganizado, el gueto presenta una organización acorde a una serie de principios en respuesta a un conjunto único de limitaciones estructurales y estratégicas que recaen en estos enclaves racializados más fuertemente que en cualquier otro segmento del territorio

41 Peterson, 1991, p. 12-13.

42 Además de los trabajos de Elijah Anderson, Mercer Sullivan, Martín Sánchez-Jankowski y Philippe Bourgois, los relatos más ilustrativos de la vida diaria en el gueto negro de hoy no son elaborados por especialistas en ciencias sociales sino por periodistas, entre ellos destacan: Sylvester Monroe y Peter Goldman con su trabajo Brothers: Black and Poor (1988), Alex Kotlowitz con There Are No Children Here (1991), Greg Donaldson con The Ville: Cops and Kids in Urban America (1993), Laurie Kay Abraham con Mama Might Be Better Off Dead (1994) y William Adler con Land of Opportunity (1995). (Honestamente, conviene recordar que los periodistas también han elaborado las representaciones más prejuiciadas, escabrosas y distorsionadas sobre los guetos). A pesar de ser citados con poca frecuencia, también cabe mencionar los trabajos de los novelistas inspirados en las urbes negras de los Estados Unidos, desde James Baldwin, Claude Brown y Piri Thomas hasta Leon Forrest y su obra Divine Days y Jess Mowry con Six Out of Seven. norteamericano ${ }^{43}$. Estas limitaciones incluyen (1) la constante presión generada por la necesidad económica y la extensa privación de material provocada por la decadencia de la economía basada en el trabajo asalariado, lo que se traduce en la desproletarización de segmentos emergentes entre la población pobre; (2) la aguda inseguridad física y social, estimulada por las notorias fallas de instituciones públicas y el correlativo debilitamiento de organizaciones locales, dando así cabida a patrones socioculturales irregulares; (3) la fuerte hostilidad racial y los agudos prejuicios de clase que desencadenan una severa y sistemática falta de oportunidades; (4) el desprestigio simbólico y la estigmatización territorial, los cuales contaminan todo 
tipo de iniciativa social, desde la amistad y la vivienda hasta los ámbitos de la educación y el trabajo; estas aversiones se ven fomentadas por (5) la apatía burocrática e ineptitud administrativa que son posibles gracias a la prescindibilidad electoral de la población negra de escasos recursos en un ambiente político dominado por lobbies corporativos e intereses financieros.

El gueto de nuestros días está constituido por un sistema social darwiniano marcado por los constantes conflictos y competencias para obtener los escasos (y menguantes) recursos en un entorno caracterizado por altos niveles de desconfianza interpersonal e institucional, una visión del mundo dominada por la competencia despiadada y altas densidades de "predadores sociales" interno se ve continuamente reforzado desde el exterior por el funcionamiento regular tanto del estado como del mercado y se mantiene estructuralmente periférico y dependiente por efecto de la fragmentación de la maquinaria político-administrativa de la metrópolis norteamericana ${ }^{45}$. La

44 Sánchez-Jankowski, 1991, especialmente p. 22-28.

45 Weiher, 1991; Weir, 1995. reconstrucción socio-analítica del habitus fracturado y de las ambiguas estrategias de "un timador" profesional del sur de Chicago revelan que la entropía característica de la vida en la calle en el corazón del gueto se encuentra modelada y obedece a una lógica social distintiva o inestable que pueden ser delineados si se asume el trabajo de relacionar meticulosamente el siempre cambiante juego de las opciones diarias con la persistente (e invisible) estructura de dominación política, económica y simbólica que predeterminan su disponibilidad, atracción y beneficios diferenciales ${ }^{46-47}$.

El cambio de la problemática de la desorganización al de la "organización" no puede reducirse a un simple cambio de terminología. Dicho proceso implica transformar el objeto a ser construido: esto significa que el experto debe explicar y detallar el modo concreto de estructuración de las relaciones y representaciones sociales -el trabajo de autoproducción colectiva- que los habitantes del gueto utilizan para dar forma, significado y propósito a su mundo y no simplemente describir en qué difiere

46 Wacquant, 1993.

47 Acerca de la racionalidad sobre la estructura social y acción en los barrios marginados véase también los análisis germinales de Portes (1972) y Perlman (1976) y sus investigaciones sobre marginalidad urbana en América Latina. En los Estados Unidos, los trabajos de Gans (1962) y Suttles (1968) merecen una segunda lectura.

revista invi № 79 / Noviembre 2013 / Volumen № 28: 165-187 177 
este modo de estructuración de aquellos que dominan otros sectores de la sociedad ${ }^{48}$. Este procedimiento conlleva demostrar cómo las actividades de las instituciones dominantes, burocracias públicas y oficinas de bienestar, escuelas y hospitales, compañías privadas y asociaciones filantrópicas, policías y agentes de libertad condicional, que se encuentren llamativamente ausentes de la ciencia social normal que aborda los barrios deprimidos (ellos brillando por su ausencia en las ciencias sociales al "interior de las ciudades", contribuyen de sobremanera a la organización del espacio social del gueto de una forma particular y especialmente desestabilizadora. Así, este proceso involucra el reconocimiento y el detalle de las bases y límites institucionales de la agencia situada de los residentes del gueto, puesto que sus prácticas y formas de vida surgen no como simples derivaciones de las constricciones que pueden ser "leídas" en las condiciones estructurales sino que sean el producto de su articulación activa con las fuerzas sociales internas y externas que componen y moldean su mundo ${ }^{49}$.

48 Hice lo posible por destacar las ganancias teóricas y empíricas que fueron posibles gracias al cambio copernicano de desorganización a organización en el caso del estudio de observación participante de Sánchez-Jankowski (1991) sobre las pandillas urbanas norteamericanas (Wacquant, 1994b).

49 Abu-Lughod et al., 1994; Bourgois, 1995.

\section{Rompiendo con el exotismo}

El concepto de desorganización ha contribuido a exotizar el gueto, en otras palabras, ha otorgado un realce artificial a patrones de conducta, sentimiento y pensamiento que difieren de una norma que supuestamente representa a la sociedad en general y de preceptos que prevalecen y son aceptados entre los propios habitantes de los guetos. De acuerdo al antropólogo sueco Ulf Hannerz ${ }^{50}$, esta firme mirada exótica tiene una larga data:

Desde los primeros días de los estudios sobre la población negra de los Estados Unidos se han discutido las formas en que los comportamientos de hombres y mujeres de raza negra-algunos hombres y mujeres en particular-difieren de aquellos de los de personas blancas.

De acuerdo a esta lógica, mientras que los habitantes más desposeídos, amenazantes y desprestigiados del centro urbano racializado representan la totalidad del gueto, los deteriorados vestigios de los cinturones negros de Norteamérica afectan e impugnan la posición cívica de toda la comunidad

50 Hannerz, 1970, p. 313. 
negra ${ }^{51}$. El resultado final es la continua reproducción de imágenes estereotipadas, superficiales y folclóricas de la población negra urbana -lo que acertadamente Ralph Ellison denominó "población negra prefabricada"- que resuenan y perpetúan en los prejuicios raciales históricos que se esconden bajo el positivismo de categorías derivadas de encuestas y el falso lenguaje neutral de la promoción de políticas.

Por esta razón, los analistas del vínculo entre raza y pobreza han prestado especial atención a las supuestas "patologías" que afectan a los habitantes de los guetos como, por ejemplo, las conductas que la llamada clase media considera anormales, ofensivas y excesivamente costosas. Estos comportamientos van desde delitos violentos, "deserción" escolar, embarazo adolescente y falta de interés laboral hasta la proliferación de "hogares encabezados por mujeres", tráfico y consumo de drogas y "dependencia del sistema de protección social". Algunos expertos no han dudado en reunir estas

51 Franklin, 1992; Fainstein, 1995. situaciones o actividades bajo el peyorativo concepto de "comportamientos de la subclase"; otros, en cambio, han ido más lejos, redefiniendo el concepto mismo de gueto como una "epidemia de problemas sociales" ${ }^{\text {"52-53 }}$

Podría demostrarse que muchas (sino la mayoría) de estas categorías, lejos de reflejar una perspectiva neutral que propicie un análisis independiente y directrices políticas imparciales, actúan como claros instrumentos de denuncia contra las conductas supuestamente anormales de los habitantes de los guetos. En este caso, se utiliza la aparentemente inofensiva expresión burocrática respecto a la "deserción" escolar, propugnada por un importante número de analistas como una variante del "comportamiento de la subclase". De igual manera, se apunta insidiosamente a los estudiantes, a sus familias "disfuncionales" y al ambiente donde ellos se desenvuelven como los responsables por el fracaso en el plano escolar cuando en realidad un buen número de las escuelas públicas los barrios

52 Jencks y Peterson, 1991: 30, 155-6, 172, 301, 322-3, 397 y pássim.

53 El trabajo de Jonathan Crane (1991) es un ejemplo caricaturesco de este ejercicio moral, el cual aparece apenas encubierto en las ciencias sociales $y$, de manera sorprendente, está presente en las páginas del American Journal of Sociology, revelando la carga político-hemilética de dicha publicación. Tal como lo demostró Sassier (1990), desde los siglos XVI al XX, los discursos sobre pobreza siempre han sido de carácter reflexivo y no estaban centrados en la condición y estado de la gente pobre sino en los conflictos políticos más importantes de cada período de acuerdo a cómo lo percibían las élites sociales e intelectuales.

revista invi № 79 / Noviembre 2013 / Volumen № 28: 165-187 
deprimidos de las grandes ciudades han sido convertidas en instituciones cuasi carcelarias que destinan más recursos a la seguridad que a la educación ${ }^{54}$ y expulsan alumnos con el fin de economizar en espacios, personal y equipamiento institucional. En este sentido, Fine ${ }^{55}$, basado en un extenso trabajo en terreno realizado en una escuela pobre de Nueva York, sugiere que "la práctica de erradicar a los 'chicos malos' es ampliamente reconocida y apreciada por directores, profesores y consejeros". Esto se debe a una buena razón: de no ser por esta medida, las escuelas enfrentarían la imposible tarea de atender a decenas de miles de estudiantes para los cuales no existe la infraestructura adecuada debido a la combinación de indiferencia política y negligencia fiscal que ha transformado a las escuelas públicas en lugares destinados a albergar niños provenientes de urbes marginadas.

Ahora bien, todo antropólogo es capaz de "identificar y describir comportamientos distintos al de su propia cultura más rápida y fielmente de lo que tiende identificar y describir aquellos

54 Devine, 1995.

55 Fine, 1988, p. 99.

180 revista invi № 79 / Noviembre 2013 / Volumen № 28: 165-187 comportamientos que le son familiares" y así "omitir o restar énfasis a los elementos pertenecientes a una cultura extranjera que presente similitudes con la suya" ${ }^{15}$. En el presente caso, la distancia social y cultural entre el analista y el objeto analizado, la escasez de observaciones de campo y la demoníaca imaginería social creada en torno al gueto se combinan para ocultar el hecho de que distintas formas de acción y organización social que pueden parecer desviadas, "aberrantes" y derechamente inexplicables desde una perspectiva externa (y superior) obedecen a una racionalidad social local adecuada a las limitaciones y facilidades del gueto negro ${ }^{57}$.

Al mismo tiempo, decir que una forma sociocultural sigue una racionalidad situada no implica necesariamente que sea específica de un lugar o grupo en particular: gran parte de las "adaptaciones" identificadas en el gueto no son propias de esta área puesto que ya han sido reconocidas en otros pueblos (sub) proletariados de Europa y América Latina y entre la población joven de origen blanco y latino de los

56 Naroll y Naroll, 1965, p. 24-26.

57 Véase la efectiva refutación de Fernandez-Kelly a la pseudo "explicación cultural" de Christopher Jencks (1991) sobre el embarazo juvenil entre la población adolescente del gueto (un postulado ad hoc al que se llega por defecto debido a la falta de un factor causal elemental; este ejercicio se ve estimulado más bien por deducciones de sentido común que por observaciones fundadas en la razón) y la devastadora crítica de Catherine Edin (1996) a la contradictoria noción de "dependencia del sistema de protección social".

ARTÍCULO: Tres premisas nocivas en el estudio del gueto norteamericano /

Loïc J.D. Wacquant 
Estados Unidos ${ }^{58}$. Esto significa, nuevamente, que la invocación de una cultura de gueto al estilo de Hannerz ${ }^{59}$ no puede sustituir un análisis empírico de las microestructuras en las que la acción social y la conciencia se encuentran enraizadas en el gueto actual. Una ironía mayor es que el estudio de Hannerz sobre los estilos de vida en el gueto de Winston Street, Washington, esconde una contradicción puesto que Soulside enfatiza la persistente diferenciación dentro de una población que ahora es homogénea al punto de carecer de identidad. De igual modo, Hannerz describe a los habitantes del gueto como esencialmente "biculturales" y reconoce que "gran parte de lo que se ha identificado como propio del gueto está directamente relacionado con la pobreza"60. Así entonces, estas características son mejor concebidas como una derivación del posicionamiento de clases (y su trayectoria pasada y probable) y no como los efectos generados por el confinamiento y la aplicación de un sistema de castas.

58 Leite Lopes, 1978; Robins y Cohen, 1978; McDermott, 1985; Foley, 1990; Jones, 1992; McLeod, 1994.

59 Hannerz, 1969.

60 Hannerz, 1969, p. 192, 182.
Para hacer frente a esta postura exótica se hace indispensable el surgimiento de una epojé moral de modo de suspender las críticas a la supuesta (in) moralidad de la vida en el gueto y enfocarse, no en las prácticas más "espectaculares" y destacadas, sino que en los intercambios y quehaceres más banales de la vida diaria y en las asumidas formas de percepción, conducta y organización que forman parte de la "realidad más importante y despierta"61 del gueto, siendo este concebido como un logro social y estratégico continuo. Ahora, afirmar que el gueto es un mundo social "significativo, razonable y normal" ${ }^{2}$ no lo glorifica ni lo vuelve más romántico. La observación etnográfica establece de forma incuestionable que el gueto es un universo gobernado por la brutalidad y la crisis, un lugar plagado de abuso, miseria y desesperanza. ¿Cómo podría ser de otra forma si el gueto es la expresión de limitaciones aplastantes y coacciones múltiples? Solo basta situar los mismos principios de análisis y

61 Schutz, 1962

62 Tal como Erving Goffman (1961, p. 7) nos hace recordar en su introducción de Internados: "Todo grupo de personas -prisioneros, primitivos, pilotos o pacientes- desarrolla una vida propia que pasa a ser significativa, razonable y normal una vez que nos acercamos a ella". Sin embargo, decir que el mundo social es "normal" no implica que aquellos que participan en él lo experimenten o acepten como tal; los campos de concentración son un ejemplo de ello. Para obtener un recuento etnográfico que muestre las formas de sociabilidad y el tejido de expresiones culturales mediante los cuales los habitantes del gueto desarrollan la "normalidad" de su mundo cotidiano a pesar del deterioro e inseguridad que los rodea, véase Wacquant (1996).

revista invi № 79 / Noviembre 2013 / Volumen № 28: 165-187 
conceptos en cualquier otro tipo de sistema social, sea este alto o bajo, glamoroso o menospreciado, familiar o ajeno, armónico o violento. Estudios sobre las atrocidades de la guerra, campos de exterminio, conflictos étnicos, recintos penitenciarios de alta seguridad o los repentinos desastres humanitarios provocados tanto por el hombre como por la naturaleza ${ }^{63}$ demuestran que, incluso en las circunstancias más extremas, la vida social es regular, basada en modelos y provista de una lógica y significados que dan cabida a elucidaciones analíticas.

Así entonces, en vez de estar centrada en la elaboración de un nuevo tipo de "orientalismo urbano" -en el sentido propuesto por Edward Said- que muestre a la "subclase" como aborrecible figura principal, la sociología debe enfocarse en desvelar la necesidad social inmanente que domina las costumbres y formas de vida de los habitantes del gueto. En definitiva, debemos tener presente el consejo de Everett C. Hughes ${ }^{64}$ en su detallada discusión sobre las "instituciones bastardas" -que tiene al gueto como ejemplo principal-:

"[ellas] deben ser estudiadas no solamente como desviaciones patológicas de lo que es bueno y correcto sino como parte de la totalidad de las actividades y emprendimientos humanos. Además, deben ser vistas como un orden de cosas que permiten observar el desarrollo de

63 Browning, 1992; Pollak, 1991; Spencer, 1990; Sykes, 1971; Erikson, 1976.

64 Hughes, 1980, p.99. procesos sociales en curso, unos procesos que, tal vez, han de encontrarse en las instituciones legitimas".

\section{Bibliografía}

ABRAHAM, L.K. Mama might be better off dead: the failure of health care in urban America. Chicago, The University of Chicago Press. 1993.

ABU-LUGHOD, J.L. Rabat: urban apartheid in Morocco. Princeton, Princeton University Press. 1980.

- et al. From urban village to east village: the battle for New York's Lower East Side. Oxford, Basil Blackwell. 1994.

ADLER, W.M. Land of opportunity: one family's quest for the American dream in the age of crack. New York, The Atlantic Monthly Press. 1995.

ANDERSON, E. Streetwise: race, class, and change in an urban community. Chicago, The University of Chicago Press. 1991.

APPADURAI, A. Putting hierarchy in its place. Cultural Anthropology 3(1): 36-49, February, 1988.

BACHELARD, G. La formation de l'esprit scientifique. Contribution à une psychanalyse de la connaissance objective. Paris, Libraire Philosophique J. Vrin. 1938.

BANFIELD, E.C. The unheavenly city. New York, Free Press. 1970 
BIDDLE, G. Alphabet city. Berkeley, University of California Press. 1992.

BOURGOIS, P. In search of respect: selling crack in El barrio. Cambridge, Cambridge University Press. 1995.

BOYER, P. Urban masses and moral order in America, 18201920. Cambridge, Harvard University Press. 1978.

BROWNING, C.R. Ordinary men: Reserve Police Battalion 101 and the final solution in Poland. New York, Harper Perennial. 1992.

BRUBAKER, W.R. Traditions of citizenship and nationhood in France and Germany. PhD dissertation, New York, Columbia University. 1991

CLARK, K.B. Dark ghetto: dilemmas of social power. New York, Harper. 1965.

CONNOLLY, H.X. A ghetto grows in Brooklyn. New York, New York University Press. 1977.

COOPERMAN, B. and CURIEL, R. The Venetian ghetto. New York, Rizzoli International Publications. 1990.

CORNELL, S. Land, labor, and group formation: Blacks and Indians in the United States. Racial and Ethnic Studies. 13(3): 253-272, 1990.

CRANE, J. The epidemic theory of ghettos and neighborhood effects on dropping out and teenage childbearing. American Journal of Sociology. 96(5): 1226-1259, March 1991.

DAVIS, M. City of quartz: excavating the future in Los Angeles. London, Verso. 1990.
DEVINE, J.A. and WRIGHT, J.D. The greatest of evils: urban poverty and the American underclass. New York, Aldine. 1993

DEVINE, J. Can metal detectors replace the panopticon? Cultural Anthropology. 10(2): 171-195, 1995.

DIMAGGIO, P. and POWELL, W.W. Introduction. En: POWELL, W.W., ed. and DIMAGGIO, P.J. ed. The new institutionalism in organizational analysis. Chicago, The University of Chicago Press.1992. p. 1-38.

DONALDSON, G. The Ville: cops and kids in urban America. New York, Tickno and Fields. 1993.

DRAKE, St. C. and CAYTON, H.R. Black metropolis: a study of Negro life in a northern city. New York, Harper and Row. 1962. 2 vols.

EDIN, K. and LEIN, L. Making ends meet: how single mothers survive welfare and low-wage work. New York, Russell Sage Foundation. 1997.

ERIKSON, K.T. Everything in its path: destruction of community in the Buffalo Creek flood. New York, Simon and Schuster. 1976

FAINSTEIN, N. Black ghettoization and social mobility. En SMITH, M., ed. and FEAGIN, J., ed. The bubbling cauldron: race, ethnicity, and the urban crisis. Minneapolis, University of Minnesota Press. 1995. p.123-141

FARLEY, R. and ALLEN, W.R. The color line and the quality of life in America. New York, Russel Sage Foundation. 1987.

revista invi № 79 / Noviembre 2013 / Volumen № 28: 165-187 
FERNANDEZ-KELLY, P. Social and cultural capital in the urban ghetto: implications for the economic sociology of immigration. En PORTES, Alejandro, ed. The economic sociology of immigration: essays on network, ethnicity, and entrepreneurship. New York, Russell Sage. 1993. p. 213-247.

FINE, M. Framing dropouts. Albany, State University of New York Press. 1988.

FISHMAN, R. Bourgeois utopias: the rise and fall of suburbia. New York, Basic Books. 1988.

FOLB, E.A. Runnin' down some lines: the language and culture of black teenagers. Cambridge, Harvard University Press. 1980.

FOLEY, D. Learning capitalist culture: deep in the heart of Texas. Philadelphia, University of Pennsylvania Press. 1990.

FRANKLIN, R. Shadows of race and class. Minneapolis, University of Minnesota Press. 1992.

FRAZIER, E.F. The Negro family in Chicago. Chicago, The University of Chicago Press. 1931.

- The Negro in the United States. New York, Macmillan. 1949.

FUSFELD, D. and BATES, T. The political economy of the ghetto. Carbondale, Southern Illinois University Press. 1984.

GANS, H. The urban villagers. New York, The Free Press. 1962.

GOFFMAN, E. Asylums: essays on the social situation of inmates. Harmondsworth, Penguin. 1961.
GOLDFIED, D.R., ed. and LANE, J.B., ed. The enduring ghetto. Philadelphia, J.B. Lippincott Company. 1973.

HANNERZ, U. Soulside: inquiries into ghetto culture and community. New York, Columbia University Press. 1969.

- What black males are like: another look. En WHITTEN, N.E., ed. and SZWED, John F., ed. AfroAmerican anthropology: contemporary perspectives, New York, The Free Press. 1970. p. 313-327.

HARRELL, A.V. and PETERSON, G.E. Drugs, crime, and social isolation: barriers to urban opportunity. Washington, DC, The Urban Institute Press. 1992.

HIRSCH, A. Making the second ghetto: race and housing in Chicago, 1940-1960. Cambridge, Cambridge University Press. 1983.

- With or without Jim Crow: black residential segregation in the United States. En HIRSCH, A., ed. and MOHL, R.A., ed. Urban policy in twentiethcentury America. New Brunswick, Rutgers University Press. 1993. p. 64-94.

HOGAN, R. The frontier as social control. Theory and Society. (14): 35-51, 1980.

HUGHES, E.C. The sociological eye. New Brunswick, Transaction. 1980

JARGOWSKI, P. Poverty and place: ghettos, barrios, and the American city. New York, Russell Sage Foundation. 1996.

- and BANE, M.J. Ghetto poverty in the United States, 1970-1980. En: JENCKS, C. and PETERSON,

ARTÍCULO: Tres premisas nocivas en el estudio del gueto norteamericano / 
P.E. The urban underclass. Washington, DC, The Brookings Institution. 1991. p. 235-273.

JAYNES, G.D. and WILLIAMS, R.M. Jr. A common destiny: blacks and American society. Washington, DC, National Academy Press. 1989.

JENCKS, C. Rethinking social policy: race, poverty, and the underclass. Cambridge, Harvard University Press. 1991.

- and PETERSON P.E., eds. The urban underclass. Washington, DC, The Brookings Institution. 1991.

JONES, J. The dispossessed: America's underclasses from the civil war to the present. New York, Basic Books. 1992.

KATZ, M. Poverty and policy in American history. New York, Academic Press. 1983.

KOTLOWITZ, A. There are no chidren here. New York, Doubleday. 1991.

KUSMER, K.L. A ghetto takes shape: Black Cleveland, 18701930. Urbana, University of Illinois Press. 1976.

LEITE LOPES, J.S. O Vapor do Diabo: O Trabalho dos Operarios do Acucar. Rio de Janeiro, Paz e Terra. 1978.

LEWIS, 0. La vida: a Puerto Rican family in the culture of poverty - San Juan and New York. New York, Random House. 1966.

LIEBOW, E. Tally's corner: a study of Negro streetcorner men. Boston, Little, Brown. 1967.

LOGAN, J.R. and MOLOTCH, H.L. Urban fortunes: the political economy of place. Berkeley, The University of California Press. 1987.
MANZA, J. Classes, status groups, and social closure: a critique of neo-Weberian social theory. Current Perspectives in Social Theory. (12): 275 302, 1992

MARKS, C. The urban underclass. Annual Review of Sociology. (17): 445-466, 1991

MASSEY, D. and DENTON, N. American apartheid: segregation and the making of the underclass. Cambridge, Harvard University Press. 1993.

MCDERMOTT, K. All dressed up and nowhere to go: youth unemployment and state policy in Britain Urban Anthropology. (14): 91-108, 1985.

MCLEOD, J. Ain't no makin' it. 2nd ed. Boulder, Westview Press. 1994

MEIER, A. and RUDWICK, E. From plantation to ghetto. New York, Hill and Wang. 1976.

MONROE, S. and GOLDMAN, P. Brothers: black and poor - a true story of courage and survival. New York, William Morrow. 1988

MOORE, J., ed. and PINDERHUGHES, R., ed. In the barrio: Latinos and the underclass debate. New York, Russell Sage Foundation. 1993.

MURPHY, R. Social closure: the theory of monopolization and exclusion. Oxford, Clarendon Press. 1988

NAROLL, R. and NAROLL, F. On bias of exotic data. Man: A Monthly Record of Anthropological Science. (63): 24-6, February, 1963.

NEWMAN, C. Culture and structure in 'The truly disadvantaged'. City and Society. 12(3): 67-83, 1991

revista invi $\mathrm{N}^{0} 79$ / Noviembre 2013 / Volumen № 28: 165-187 
O'CONNOR, Alice Race and class in Chicago sociology. Paper presented to the Social Science History Association Meetings. November, 1995

OSOFSKY, G. Harlem: the making of a ghetto - Negro New York, 1890-1930. 2nd ed. New York, Harper. 1971.

PARK, R.E. and BURGESS, W. The city. Chicago, The University of Chicago Press. 1925.

PARKIN, F. Marxism and class theory: a bourgeois critique. New York, Columbia University Press. 1978.

PATTERSON, J. America's struggle against poverty. 2nd ed. Cambridge, Harvard University Press. 1986.

PERLMAN, J. The myth of marginality. Berkeley, The University of California Press. 1976.

PETERSON, P. The urban underclass and the poverty paradox. En JENCKS, C., ed. and PETERSON, P.E., ed. The urban underclass. Washington, DC, The Brookings Institution. 1991. p. 3-27.

POLLAK, M. L'experience concentrationnaire. Paris, A.-M. Metaille. 1991.

PORTES, A. Rationality in the slum: an essay in interpretative sociology. Comparative Studies in Society and History. 14(3): 268-86, June 1972.

RAINWATER, L. Behind ghetto walls: black families in a federal slum. Chicago, Aldine. 1970.

ROBINS, D. and COHEN, P. Knuckle sandwich: growing up in the working-class city. Harmondsworth, Penguin Books. 1978.
SÁNCHEZ-JANKOWSKI, M. Islands in the street: gangs in urban American society. Berkeley, University of California Press. 1991.

SASSIER, Philippe. Du bon usage des pauvres : histoire d'un theme politique (XVIe-XXe siecle). Paris, Fayard. 1990.

SCHUTZ, A. Collected papers I: the problem of social reality. The Hague, Martinus Nijhoff. 1962.

SHAW, C. and MCKAY, H. Juvenile delinquency in urban areas. Chicago, The University of Chicago Press. 1942.

SNIPP, C.M. The changing political and economic status of American Indians: from captive nations to internal colonies. American Journal of Economics and Sociology. 45(2): 145-157, April 1986.

SPEAR, A.H. Black Chicago: the making of a Negro ghetto, 1890-1920. Chicago, The University of Chicago Press. 1968.

SPENCER, J. Collective violence and everyday practice in Sri Lanka. Modern Asian Studies. 24(3): 603-623, 1990.

SUTTLES, G.D. The social order of the slum: ethnicity and territory in the inner city. Chicago, The University of Chicago Press. 1968.

SYKES, G.M. The society of captives: a study of a maximum security prison. Princeton, Princeton University Press. 1971, orig. 1958.

TROTTER, W.J., Jr. Blacks in the urban north: the 'underclass question' in historical perspective. En: KATZ, M.B., ed. The underclass debate: views

ARTÍCULO: Tres premisas nocivas en el estudio del gueto norteamericano / Loïc J.D. Wacquan 
from history. Princeton, Princeton University Press. 1993. p. 55-81.

VERGARA, C. The new American ghetto. New Brunswick, Rutgers University Press. 1995.

WACQUANT, L.J.D. What makes a ghetto? Notes toward a comparative analysis of modes of urban exclusion. Paper presented at the Working Conference on Poverty, Immigration and Urban Marginality in Advanced Societies. Paris, Maison Suger, 10-11 May, 1991

- 'The zone': le metier de 'hustler' dans le ghetto noir americain. En BOURDIEU, Pierre et al. La misere du monde. Paris, Editions du Seuil. 1993. p. 181-204. [Traducido como 'Inside the zone'. En BOURDIEU, P. et al. The poverty of society. Cambridge Polity Press. 1997].

- The new urban color line: the state and fate of the ghetto in postfordist America. En Calhoun, C.J., ed. Social theory and the politics of identity. Oxford, Basil Blackwell. 1994a. p. 231-276.

- Le gang comme predateur collectif. Actes de la recherche en sciences sociales. (101-102): 88-100, March 1994b.

- Elementary forms of racial domination. Rockefeller Lecture presented to the Nucleo da Cor, Instituto de Filosofia e Ciencias Sociais, Universidade federal do Rio de Janeiro, Brazil. October 10, 1995.

- Un mariage dans le ghetto. Actes de la Recherche en Sciences Sociales. (113): 63-84, June 1996.

WARD, D. Poverty, ethnicity, and the American city, 1840-1925. Cambridge, Cambridge University Press. 1989.
WEAVER, R. The Negro ghetto. New York, Russell and Russell. 1948.

WEBER, M. Economy and society. Berkeley, University of California Press. 1978.

WEIHER, G. The fractured metropolis: political fragmentation and metropolitan segregation. Albany, State University of New York Press. 1991.

WEIR, M. The Politics of racial isolation in Europe and America. En PETERSON, P.E. ed. Classifying by race. Princeton, Princeton University Press. 1995. p. 217-242.

WHYTE, W.F. Street corner society: the social structure of an Italian slum. Chicago, The University of Chicago Press. 1943.

WILSON, W.J. The truly disadvantaged: the inner city, the underclass and public policy. Chicago, The University of Chicago Press. 1987.

- and APONTE, R. Urban Poverty. Annual Review of Sociology. (11): 231-58, 1985.

WIRTH, L. The ghetto. Chicago, The University of Chicago Press. 1928.

- On cities and social life. Edited and with an introduction by Albert J. Reiss, Jr. Chicago, The University of Chicago Press. 1964.

WITTGENSTEIN, L. Vermischte Bermerkungen. Frankfurt, Suhrkamp. 1977.

ZUNZ, 0. The changing face of inequality: urbanization, industrial development, and immigrants in Detroit, 1880-1920. Chicago, The University of Chicago Press. 1982.

revista invi № 79 / Noviembre 2013 / Volumen № 28: 165-187 